\title{
An experimental study of the combined effects of $n$-hexane and methyl ethyl ketone
}

\author{
Y TAKEUCHI, ${ }^{1} \mathrm{Y}$ ONO,${ }^{1} \mathrm{~N}$ HISANAGA,${ }^{1} \mathrm{M}$ IWATA,${ }^{1} \mathrm{M}$ AOYAMA,${ }^{1} \mathrm{~J}$ KITOH,${ }^{2}$ \\ AND Y SUGIURA ${ }^{2}$
}

From the Departments of Hygiene ${ }^{1}$ and Anatomy, ${ }^{2}$ Nagoya University School of Medicine, Nagoya, Japan

\begin{abstract}
This study was intended to determine whether or not methyl ethyl ketone (MEK) enhances the neurotoxicity of $n$-hexane at low concentration and after long term exposure. Separate groups of eight rats were exposed to $100 \mathrm{ppm} n$-hexane, $200 \mathrm{ppm}$ MEK, $100 \mathrm{ppm}$ $n$-hexane plus $200 \mathrm{ppm}$ MEK, or fresh air in an exposure chamber for 12 hours a day for 24 weeks. The body weight, motor nerve conduction velocity (MCV), distal motor latency (DL), and mixed nerve conduction velocities (MNCVs) were measured before exposure and after four, eight, 12, 16, 20, and 24 weeks' exposure. One rat of each group was histopathologically examined after 24 weeks' exposure. Exposure of $100 \mathrm{ppm} n$-hexane did not significantly decrease the functions of the peripheral nerve throughout the experiment. Exposure to $200 \mathrm{ppm} \mathrm{MEK}$ significantly increased MCV and MNCVs and decreased DL after four weeks' exposure, but at the later stage no significant changes were found throughout the experiment by comparison with the controls. Mixed exposure to $100 \mathrm{ppm} n$-hexane plus $200 \mathrm{ppm}$ MEK significantly decreased DL after four weeks' exposure and decreased MCV and MNCVs after 20 and 24 weeks' exposure by comparison with the controls. On histopathological examination of the tail nerve, however, no changes were found in any of the exposed groups or the controls. These results suggest that MEK might enhance the neurotoxicity of $n$-hexane at a low concentration, and mixed exposures to $n$-hexane and MEK should be avoided.
\end{abstract}

$n$-Hexane is widely used in industry and is well known to be neurotoxic. It is often used with other solvents; and, as a result, many workers are exposed to mixed organic solvents containing $n$-hexane. ${ }^{12}$ The study of the combined effects of $n$-hexane and other solvents on the nervous system is important for the maintenance of the health of men exposed at work. Billmaire $e t a l^{3}$ and Allen $e t a^{4}$ reported an outbreak of peripheral neuropathy in workers exposed to methyl $n$-butyl ketone (MBK) (a metabolite of $n$-hexane) and methyl ethyl ketone (MEK) in a coated fabrics plant. Saida $e t a^{5}$ reported that MEK did not produce peripheral neuropathy in the rat, but the combination of MBK and MEK did. Altenkirch and Mager ${ }^{6}$ reported a polyneuropathy in four youngsters who sniffed thinners containing $n$-hexane, MEK, and other solvents. Altenkirch et al also showed in an experimental study, ${ }^{7}$ that exposure to $1000 \mathrm{ppm}$ MEK plus 9000

Received 24 May 1982 Accepted 23 July 1982 ppm $n$-hexane shortened the onset of severe polyneuropathy compared with exposure to 10000 ppm $n$-hexane. On the other hand, Takeuchi $e^{2} a l^{8}$ showed that $1000 \mathrm{ppm}$ toluene inhibited the neurotoxicity of $1000 \mathrm{ppm} n$-hexane. Ono et al ${ }^{9}$ showed that even $200 \mathrm{ppm} n$-hexane could affect the peripheral nerve in rats. Therefore, in the present study we intended to clarify whether or not MEK could increase the neurotoxicity of $n$-hexane in the rat, even at the low concentrations used in industry, and whether $100 \mathrm{ppm} n$-hexane alone could affect the peripheral nerves.

\section{Material and methods}

Thirty two Wistar strain male rats (mean body weight $318 \mathrm{~g}, \mathrm{SD} \pm 10 \mathrm{~g}$ ) were divided into four groups of eight rats, and the separate groups were exposed to $100 \mathrm{ppm} n$-hexane, $200 \mathrm{ppm}$ MEK, 100 $\operatorname{ppm} n$-hexane plus $200 \mathrm{ppm}$ MEK, or fresh air in an exposure chamber ${ }^{10}$ for 12 hours a day, every day for 24 weeks. Food and water were provided freely. 
The temperature and the relative humidity in the chambers fluctuated from $23-24^{\circ} \mathrm{C}$ and $40-60 \%$. The $n$-hexane and MEK used were more than $99 \%$ pure, and the concentrations of vapour in the exposure chamber were measured daily by gas chromatography. The measured concentrations were $106 \pm 35 \mathrm{ppm}$ of $n$-hexane, $200 \pm 40 \mathrm{ppm}$ of MEK, and $104 \pm 26 \mathrm{ppm}$ of $n$-hexane plus $203 \pm 26$ ppm of MEK (mean \pm SD, for 24 weeks). The peripheral nerve conduction velocity was measured in the tail nerve of the rat by the methods previously reported. ${ }^{81112}$ The rat was wrapped in a towel to keep it immobilised without anaesthesia and laid on its back to allow electrodes to be inserted in the ventral part of the tail. The electrode was a stainless steel needle, $0.34 \mathrm{~mm}$ in diameter and about $15 \mathrm{~mm}$ long. Electrode $\mathrm{A}$ was inserted $3 \mathrm{~cm}$ distal from the anus, electrode $\mathrm{C}, 3-4 \mathrm{~cm}$ proximal to the end of the tail, and electrode $B, 5 \mathrm{~cm}$ proximal to $C$. The references were inserted about $2 \mathrm{~mm}$ from each electrode, and the body earth was inserted between B and C. After insertion of the electrodes, the tail was immersed in a paraffin bath in which the temperature was maintained between $37^{\circ}$ and $38^{\circ} \mathrm{C}$. The conduction velocity of the tail nerve was measured more than four minutes after the immersion, and the measurement was finished within 20 minutes of the immersion. The tail nerve was stimulated by a square pulse of $0.3 \mathrm{msec}$ duration, $2 \mathrm{c} / \mathrm{s}$ and supramaximal strength with an electrostimulator (SEN-7103, Nihon Koden), and the biopotentials were observed with an Addscope (ATAC-350, Nihon Koden). Points A and B were stimulated by a single stimulation and the muscle action potentials were observed at point $C$ to obtain motor nerve conduction velocity (MCV) and the motor distal latency (DL). The tail nerve was stimulated 100 times at point $\mathrm{C}$, and the action potentials at points $A$ and $B$ were summated to obtain mixed nerve conduction velocities (MNCVs). The formulae of these indices are as follows: $\mathrm{MCV}=$ distance $(\mathrm{AB}) /$ latency time (AC-BC), DL = latency time (BC), $\mathrm{MNCV}(\mathrm{CB})($ distal $)=$ distance $(\mathrm{CB}=5 \mathrm{~cm}) /$ latency time $(\mathrm{CB}), \mathrm{MNCV}(\mathrm{CA})$ (total) = distance (CA)/latency time (CA), and MNCV (BA) (proximal) $=$ distance (BA)/latency time (CA-CB). The body weight, MCV, DL, and MNCVs were measured before exposure and every four weeks after exposure.

One rat of each exposed group and one control animal were killed after 24 weeks' exposure and pathohistologically studied. Under anaesthesia, the rat was perfused from the left ventricle with a fixative that contained paraformaldehyde and glutaraldehyde. About $1 \mathrm{~cm}$ of nerve tissue was dissected out from the proximal and distal portions of the dorsal tail nerve trunk. For light microscopic examination of the teased nerve, nerve tissues were postfixed in osmium tetroxide and dehydrated in glycerin, and teased by a needle in glycerin under binocluar dissection microscope. For electron microscopic examination, tissues were fixed in the same fixative and then postfixed with osmium tetroxide. After staining en bloc with uranyl acetate, the tissues were dehydrated and embedded in epon. Ultrathin sections were stained with uranyl acetate and lead citrate and examined with an electron microscope (JEOL $100 \mathrm{CX}$ ). The present electrophysiological results were compared with those from the previous 200 and $500 \mathrm{ppm} n$-hexane exposure. 9

\section{Results}

The changes in body weight, MCV, and DL are shown in fig 1 , and the changes in the MNCVs in fig 2 . The body weight of the rats in the exposed groups showed no significant differences from the controls throughout the experiment. No abnormal behaviour was observed in either the exposed groups or the controls throughout the experiment. In the $200 \mathrm{ppm}$ MEK group a significant increase in MCV and
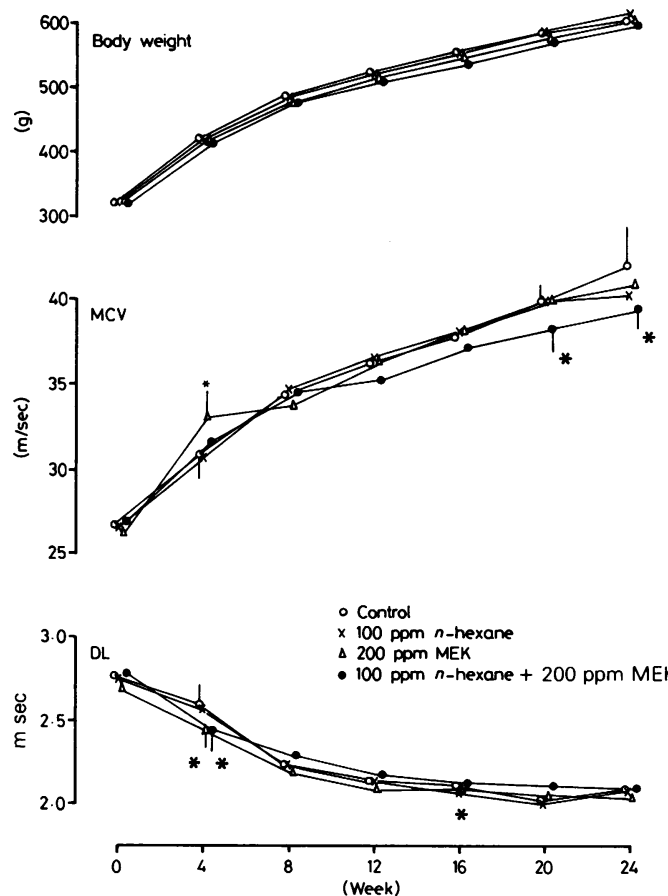

Fig 1 Changes in body weight, $M C V$, and $D L$. Significance level: ${ }^{*} p<0.05$. 

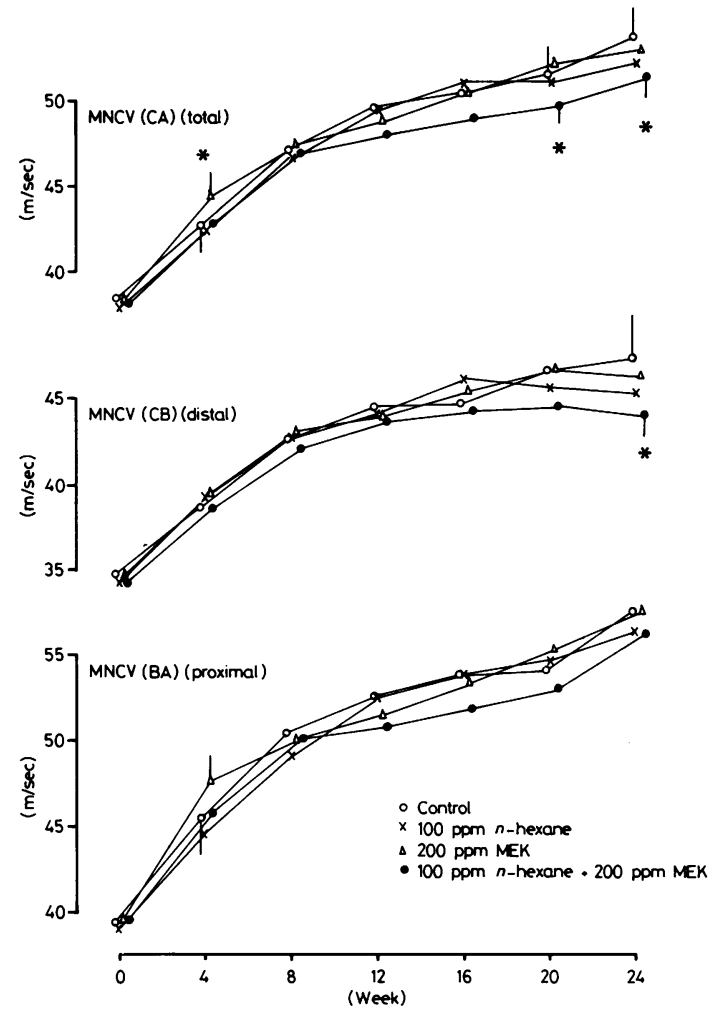

Fig 2 Changes in MNCVs. Significance level: *(week) $p<0.05$.
MNCV (total), and a significant decrease in DL were shown by comparison with the controls after four weeks' exposure, but at the later stage no significant differences were found throughout the experiment. In the $100 \mathrm{ppm} n$-hexane group a significant decrease in DL after four weeks' exposure and a tendency for MNCV (total) to decrease after 24 weeks' exposure was shown, but no other significant differences were found in any electrophysiological index by comparison with the controls. In the $100 \mathrm{ppm} n$-hexane plus $200 \mathrm{ppm} \mathrm{MEK}$ group a significant decrease in MCV, and MNCVs (total and distal) after 20 and 24 weeks' exposure was shown by comparison with the controls. A significant increase in DL and a significant decrease in MNCVs (total and proximal) were found after 16 weeks' exposure in the mixed exposure group by comparison with the group of $n$-hexane alone; no other significant differences were found between the two groups. A significant increase in MNCV (total) was found in the mixed exposure group by comparison with the group of MEK alone after four weeks' exposure; ir: zther significant differences were found. The results of the electrophysiological examinations are summarised in the table.

The changes in MCV of the present $100 \mathrm{ppm}$ $n$-hexane, $200 \mathrm{ppm}$ MEK, and $100 \mathrm{ppm} n$-hexane plus $200 \mathrm{ppm}$ MEK, and the previous $200 \mathrm{ppm}$ and $500 \mathrm{ppm} n$-hexane groups ${ }^{9}$ are shown in fig 3 . A dose-dependent effect of $n$-hexane on the function of the peripheral nerve is found in 100,200 , and 500 $\mathrm{ppm} n$-hexane exposure, and the effects of $100 \mathrm{ppm}$ $n$-hexane plus $200 \mathrm{ppm}$ MEK are found between the

Summary of significant differences found in neurophysiological values in exposed and control animals

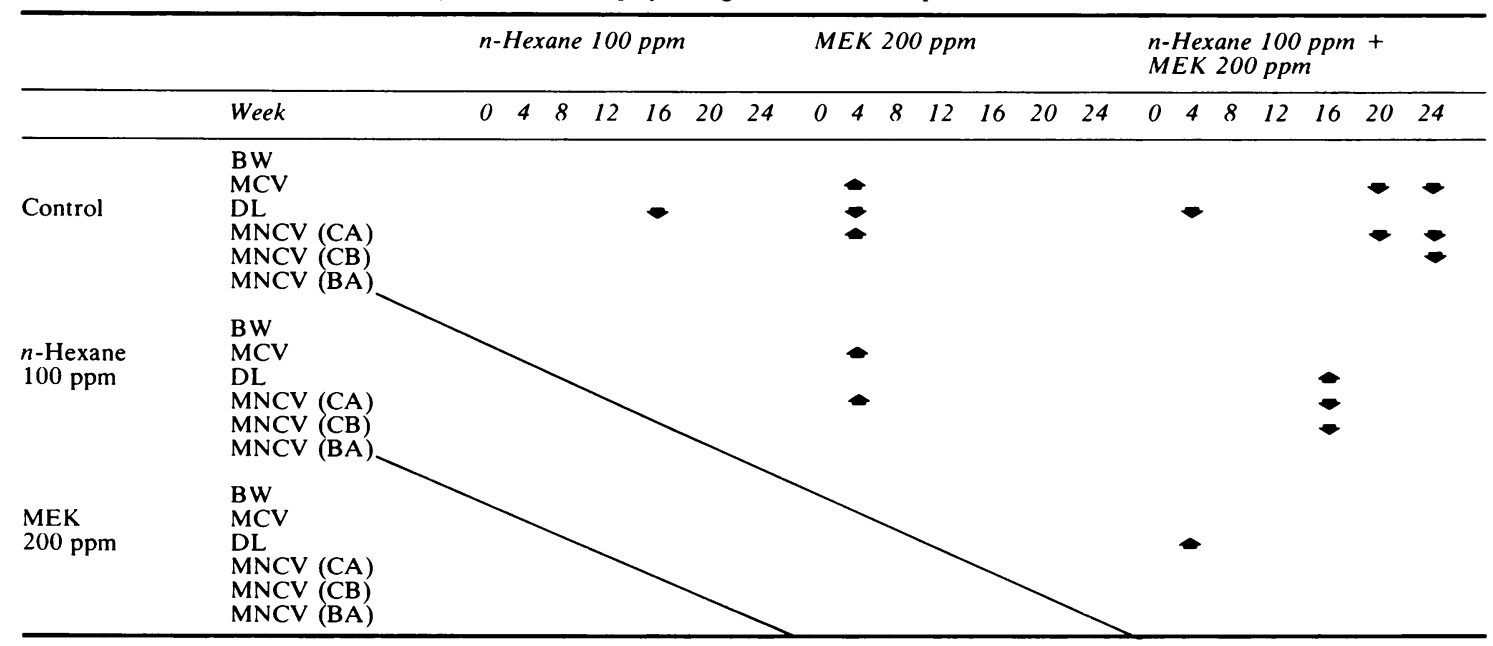

Arrows show statistically significant level $(p<0 \cdot 05)$ and directions.

$\mathrm{BW}=$ Body weight; $\mathrm{MCV}=$ Motor nerve conduction velocity; $\mathrm{DL}=$ Distal motor latency; $\mathrm{MNCV}=$ Mixed nerve conduction velocity. 


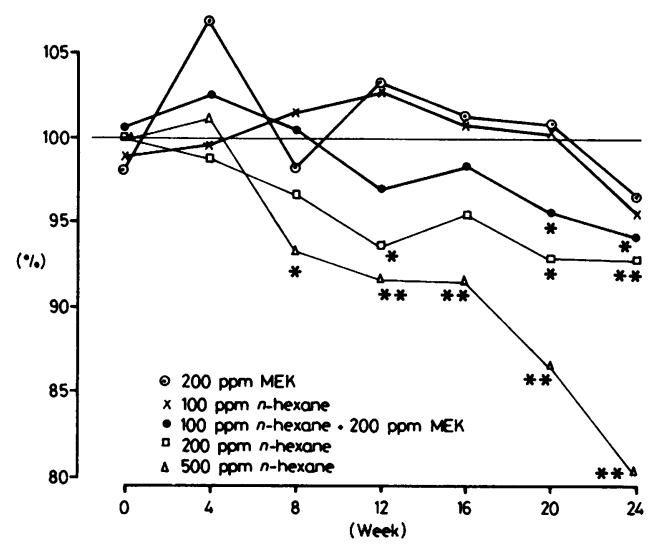

Fig 3 Changes in MCV (percentage of the exposed groups in the controls). Significance level: ${ }^{*} p<0.05,{ }^{* *} p<0.01$.

exposure of $100 \mathrm{ppm}$ and $200 \mathrm{ppm} n$-hexane. Microscopic examination of the teased nerves showed no changes in the tail nerves of rats in the present $100 \mathrm{ppm} n$-hexane, $200 \mathrm{ppm}$ MEK, 100 ppm $n$-hexane plus $200 \mathrm{ppm}$ MEK groups, or the controls.

\section{Discussion}

The present study showed that exposure to $100 \mathrm{ppm}$ $n$-hexane plus $200 \mathrm{ppm}$ MEK impaired the function of the peripheral nerve in the rat and that exposure to $100 \mathrm{ppm} n$-hexane alone did not. The differences between the mixed exposure group and the $100 \mathrm{ppm}$ $n$-hexane group were not as clear as those that Altenkirch $e t a^{7}$ showed at high concentrations. These results suggest that mixed exposure to $n$-hexane and MEK might be more toxic than $n$-hexane alone even at the low concentrations used in industry, and that exposure to this mixture should be avoided.

The present experiment showed no significant changes in the $100 \mathrm{ppm} n$-hexane group except for DL after 16 weeks' exposure by comparison with the controls throughout the experiment, although MNCV (CA) (total) showed a tendency to decrease after 24 weeks. On the other hand, Ono et $a l^{9}$ and Kitoh et $a^{13}$ reported that exposure to $200 \mathrm{ppm}$ $n$-hexane affected the peripheral nerve of the rat in conditions similar to those of the present experiment, and that changes could be detected by both electrophysiological and histopathological examination. The present histopathological examinations of the tail nerve trunks showed no changes in either the exposed groups or in the controls. From these results it might be inferred that the changes of the peripheral nerve in rats can be detected earlier by electrophysiological examination than by histopathological examination. And as the dose of 100 $\operatorname{ppm} n$-hexane for 12 hours a day every day is equivalent to that of $175 \mathrm{ppm}$ for eight hours a day, six days a week, it might also be inferred that the present electrophysiological methods can detect impairment of the function of the peripheral nerve in rats exposed to the concentration between 175 to $350 \mathrm{ppm}$ of $n$-hexane for eight hours a day, six days a week for 24 weeks. Sanagi et al $^{14}$ reported that workers exposed to $58 \pm 41 \mathrm{ppm}$ (time weighted average for eight hours) $n$-hexane developed impairment of the nerve conduction velocities. And, according to the previous review of the doseresponse relationship of $n$-hexane, ${ }^{1}$ it can be speculated that workers exposed to about $600 \mathrm{ppm}$ (TWA for eight hours) $n$-hexane could develop severe polyneuropathy with muscle atrophy and gait disturbance, whereas it would require an exposure of about $4700 \mathrm{ppm}$ (TWA for eight hours) $n$-hexane for rats to develop the same. Judging from these results, it may be inferred that man is several times as sensitive to $n$-hexane as rats at both low and high concentrations.

In the $200 \mathrm{ppm}$ MEK exposure group the MCV and MNCVs significantly increased and DL significantly decreased by comparison with the controls after four weeks' exposure, but these changes were not found at the later stage of the experiment. Savolainen and Seppäläinen ${ }^{15}$ reported that a significant increase in MCV and in axonal membrane protein was noted in rats exposed to $300 \mathrm{ppm}$ toluene for three weeks by comparison with the controls, and that these changes could not be found at the later stage. These results suggest that the 200 ppm MEK exposure might also affect the axonal membrane and transiently increase the nerve conduction velocity similar to $300 \mathrm{ppm}$ toluene at the earlier stage of exposure, although the chronic mixed effects of MEK and $n$-hexane are opposite to those of toluene and $n$-hexane.$^{8}$ The mechanism by which MEK enhances the neurotoxicity of $n$-hexane is interesting but, as yet, not clear. Abdel-Rahman et $a l^{16}$ reported that the combined exposure of 225 ppm MBK and $750 \mathrm{ppm}$ MEK enhanced the neurotoxicity of MBK and increased MBK and 2, 5 -hexanedione (a common metabolite of $n$-hexane and MBK) in the blood of rats by comparison with $225 \mathrm{ppm}$ MBK alone. Couri et al ${ }^{17}$ reported that the combined administration of MBK/MEK (40/120 mg ip) increased the urinary excretion of 2-hexanol and 2, 5-hexanedione compared with the administration of an equal dose of MBK alone. These results suggest that MEK itself is not toxic to the peripheral nerve but that it might modify the biotransformation of, and enhance the neurotoxicity of, $n$-hexane. 


\section{References}

' Takeuchi Y, Hisanaga N, Ono Y, Inoue T. Toxicity and doseresponse (effect) relationship of $n$-hexane. Jpn J Ind Health 1980;22:470-87.

2 Spencer PS, Schaumburg HH, Sabri MI, Veronesi B. The enlarging view of hexacarbon neurotoxicity. CRC Crit Rev Toxicol 1980;7:279-356.

${ }^{3}$ Billmaire DJ, Yee HT, Allen N, Craft B, Epstein S, Fontaine RE. Peripheral neuropathy in a coated fabrics plant. JOM 1974;16:665-71.

${ }^{4}$ Allen N, Mendell JR, Billmaire DJ, Fontaine RE, O'Neill J. Toxic polyneuropathy due to methyl n-butyl ketone. Arch Neurol 1975;32:209-18.

${ }^{5}$ Saida K, Mendell JR, Weiss HS. Peripheral nerve changes induced by methyl n-butyl ketone and potentiation by methyl ethyl ketone. J Neuropaih Neurol 1976;35:207-25.

- Altenkirch H, Mager J. Toxische Polyneuropathien durch Schnuffeln von Pattex-Verdunner. Dtsch Med Wochenschr 1976;6:195-9.

${ }^{7}$ Altenkirch H, Stoltenburg G, Wagner HM. Experimental studies on hydrocarbon neuropathies induced by methyl ethyl ketone (MEK). J Neurol 1978;219:159-70.

' Takeuchi Y, Ono Y, Hisanaga N. An experimental study on the combined effects of $n$-hexane and toluene on the peripheral nerve of the rat. $\mathrm{Br} J$ Ind Med 1981;38:14-9.

- Ono Y, Takeuchi Y, Hisanaga N, Kitoh J, Sugiura Y. Neurotoxicity of petroleum benzine compared with $n$-hexane. Int Arch Occup Environ Health 1982;50:219-29.
"Maeda K. Gas chambers for the exposure of animals to organic solvents. Jpn J Ind Health 1968;10:427-32.*

"Ono Y, Takeuchi Y, Hisanaga N. Studies on methods to measure nerve conduction velocity in rat's tail and comparative toxicity of $n$-hexane, methyl n-butyl ketone and 2,5-hexanedione. Jpn $J$ Ind Health 1979;21:528-38. ${ }^{*}$

1: Takeuchi Y, Ono Y, Hisanaga N, Kitoh J, Sugiura Y. A comparative study on the neurotoxicity of $n$-pentane, $n$-hexane and $n$-heptane in the rat. BrJ Ind Med 1980;37:241-7.

${ }^{13}$ Kitoh J, Sugiura Y, Ono Y, Hisanaga N, Takeuchi Y. A histopathological study on the neurotoxicity of $n$-hexane and petroleum benzine. Proceedings of the 54th annual meeting of Japanese Association of Industrial Health 1981. Japan Association of Industrial Health, 1981:180-1. (In Japanese.)

${ }^{14}$ Sanagi S, Seki Y, Sugimoto K, Hirata M. Peripheral nervous system functions of workers exposed to $n$-hexane at a low level. Int Arch Occup Environ Health 1980;47:69-79.

is Savolainen H, Seppäläinen AM. Biochemical and physiological effects of organic solvents on rat axon membranes isolated by a new technique. Neurotoxicology 1980;1:464-77.

16 Abdel-Rahman MS, Hetland LB, Couri D. Toxicity and metabolism of methyl n-butyl ketone. Am Ind Hyg Assoc J 1976;37:95-102.

1 Couri D, Abdel-Rahman MS, Hetland LB. Biotransformation of $n$-hexane and methyl $n$-butyl ketone in guinea pigs and mice. Am Ind Hyg Assoc J 1978;39:295-300.

${ }^{*}$ In Japanese with English abstract. 